\title{
CONFLICTING INTERESTS, DECOMPOSABILITY, AND COMPARATIVE STATICS
}

\author{
Mark WALKER \\ Department of Economics, SUNY Stony Brook, Stony Brook, NY 11794, U.S.A. \\ Communicated by $\mathrm{H}$. Moulin \\ Received 6 September 1988 \\ Revised 12 December 1988
}

\begin{abstract}
When optimizing an aggregate of several individual objective functions, it may be possible to decompose the set of individual objectives into groups across which there are no conflicting interests. It is shown that changes in an individual objective will affect those individuals, and only those individuals, whose objective is in potential conflict with the changed objective. Thus, in particular, each individual can affect every other individual if and only if the optimization problem is indecomposable - i.e. if and only if it is impossible to separate the individuals into groups across which there are no conflicting interests.
\end{abstract}

Key words: Decomposability; sensitivity analysis; aggregate optimization; comparative statics.

\section{Introduction}

Consider a situation in which an action, or program, must be chosen, and suppose that each of the available alternative programs has several real components. In other words, each alternative program is a list of, say, $m$ real numbers. In attempting to choose the best program, suppose that our goal is to maximize the total utility (for example, the total profit, revenue, sales, etc.) of a group of individual units, or agents, where the utility of each one of the agents is affected by the program that we choose. Suppose further that each agent's utility is un affected by changing the program in certain ways - each agent has a certain indifference about alternative programs. The question that I wish to explore in this paper is whether there is a useful relation between the structure of these 'indifferences' - i.e. the structure of the decision problem - on the one hand, and, on the other hand, the effects that a change in one agent's utility function will have upon the other agents' utility levels. In other words, can the structure of a collective decision problem, or an aggregate optimization problem, have useful implications for the inter-agent sensitivity analysis, or the comparative statics, of the problem?

Even at this early stage, it will be helpful to have a bit of notation. Let us represent programs by vectors $x \in \mathbb{R}^{m}$; let us index the agents by the elements of the set $N=\{1,2, \ldots, n\}$; and let us characterize each agent $i \in N$ by his utility function 
$u_{i}: \mathbb{R}^{m} \rightarrow \mathbb{R}$, which describes how his utility depends upon the program that is chosen.

We are interested in the way that the optimal program depends upon the individuals' utility functions. We will assume throughout that the utility functions are sufficiently well-behaved that for any profile $u=\left(u_{1}, \ldots, u_{n}\right)$ of utility functions there is a unique optimal program and that the optimal program varies smoothly with changes in the functions $u_{i}$. Let us denote this smooth function from profiles to programs by $\pi: u \mapsto x$. At this level of generality the analysis of the differential properties of the function $\pi$ is well understood: it is a straightforward application of the Implicit Function Theorem.

In many applications it is important to take account of certain additional structure that the problem exhibits. In particular, we often know that each agent's utility does not depend upon all $m$ components of a program, but only on a (fixed) subset of components. More generally, an agent's utility may depends only upon a set of 'generalized' components - i.e. each agent $i \in N$ may be characterized by a subspace $I_{i}$ of $\mathbb{R}^{m}$, in the sense that his utility level $u_{i}(x)$ will be unaffected by varying the program $x$ if the variation lies in the subspace $I_{i}$. We refer to $I_{i}$ as $i$ 's 'indifference space' and to its orthogonal complement (denoted $A_{i}$ ) as his 'affective space'.

A classical example of the situation we have described so far is an 'exchange economy', in which there are $k$ 'commoditites' and each agent cares only about how much of each commodity is allocated to him. A program is an $n k$-tuple (i.e. $m=n k$ ), and we can order the components in such a way that agent 1 cares about the first $k$ (i.e. $A_{1}$ is the subspace spanned by the first $k$ unit vectors), agent 2 cares about the next $k$, and so on. Notice that in this example the economy is 'decomposable' in the sense that $\mathbb{R}^{m}$ is the direct sum of the spaces $A_{i}$ - i.e. every vector in $\mathbb{R}^{m}$ can be expressed in a unique way as a sum $\sum x_{i}$, where each $x_{i} \in A_{i}$.

A second example is a variation of the first one: suppose that the total amount of each commodity $k$ that will be allocated to the $n$ agents is a fixed number of units. Now the set of possible (or feasible) allocations is an $(n-1) k$-dimensional affine subspace of $\mathbb{R}^{n k}$, or more conveniently, simply $\mathbb{R}^{m}$, where $m=(n-1) k$. Now if we consider each individual's utility for feasible allocations, it is no longer possible, as it was in the first example, to decompose the economy into disjoint sets $N_{p}$ of agents and 'corresponding' subspaces $Z_{p}$ of the feasible space - i.e. subspaces $Z_{p}$ whose direct sum is $\mathbb{R}^{m}$ and that have the property that each $A_{i}$ is a subspace of the space $Z_{p}$ for which $i \in N_{p}$. In this example, then, it seems as if we ought to say that the economy is 'indecomposable'.

The second example is of course the only one that is really interesting. In the first example the decompositon of the economy corresponds in an obvious way to a decomposition of the optimization problem: we can find the optimal program $x$ by simply choosing, for each $i$, the $x_{i}$ (or the appropriate projection of $x_{i}$ ) that maximizes $u_{i}$. In the second example, the optimization problem cannot be decomposed because it takes explicit account of the conflicting interests of the agents, and it is these conflicting interests that cannot be decomposed.

It turns out that there is another way in which the two examples differ from one 
another. In the first example it is clear that if we change one of the functions $u_{i}$ (but change it in such a way that agent $i$ still cares only about the quantities allocated to himself), then the resulting change in the optimal program $x=\pi(u)$ will not affect any other agent's utility level. In the second example, on the other hand, the utility level of every agent can be affected (at least generically) by changing the slope of any other agent's utility function - every agent can affect every other agent in the second example. This property is an important one in general, not just in this example, and it seems to be related to the indecomposability of the optimization problem.

The remainder of the paper is devoted to an analysis of the relation between the two ideas of decomposition and 'affectability'. It will be shown that in fact the two ideas are equivalent: in an optimization problem of the kind we have outlined, a pair of agents cannot affect one another if and only if they can be separated -i.e. if and only if there is a decomposition of the problem which leaves the two agents in separate component parts of the decomposition. Thus, in particular, the second example is not misleading: in any indecomposable optimization problem (that is, in any problem in which the conflicting interests of the agents cannot be decomposed), all of the agents will be able to affect one another.

The theory that will be developed here has been applied elsewhere to an important problem in economic theory, namely the question of whether there are allocation mechanisms that (a) are immune to manipulation by the agents participating in them and (b) always achieve allocations that are Pareto optimal. Hurwicz and Walker (1988) have shown that an allocation mechanism cannot satisfy both (a) and (b) if all participants are able to affect one another in the sense outlined in the preceding paragraphs. The results in the present paper, then, support a much simpler characterization of the 'bad' economies in Hurwicz and Walker (1989): if an economy is indecomposable - i.e. if the participants cannot be separated into groups across which there are no conflicting interests - then the economy will not admit a 'successful' allocation mechanism (one that satisfies both (a) and (b)).

The remainder of the paper is organized as follows: Section 1 defines the structure of decision problems in terms of the agents' affective and indifference spaces, and defines the notion that one agent can affect another agent. Sections 2 and 3 define the notion of a decomposition of an affective-space structure and develop a useful characterization of decomposable structures. The main result - that the presence or absence of inter-agent effects can be characterized by the decomposability of the affective-space structure of the decision problem - is established in Section 4. A concluding section describes a potential application to the economic problem of externalities and decentralization.

\section{The affective structure of a decision problem}

Since all of our analysis will concern first-order properties of the function $\pi$, the analysis will be conducted in terms of the Hessian matrices (matrices of second par- 
tial derivatives) of the functions $u_{i}$. Consequently, nothing will be lost if we assume that the utility functions are all quadratic:

$$
u_{i}(x)=\frac{1}{2} x^{\prime} Q^{i} x+b_{i}^{\prime} x .
$$

The first derivative (the gradient) at a vector $x$ is therefore

$$
\nabla u_{i}(x)=\left[\frac{\partial u_{i}}{\partial x_{k}}\right]_{k=1}^{m}=Q^{i} x+b_{i},
$$

and the matrix of second partial derivatives at every $x$ is just $Q^{i}$ :

$$
\left[\frac{\partial^{2} u_{i}}{\partial x_{r} \partial x_{s}}\right]=Q^{i}
$$

(Matrices will be indexed with superscripts in order to enable us to index their elements with subscripts.)

For each profile $u=\left(u_{1}, \ldots, u_{n}\right)$ we denote the aggregate objective function by $\varphi_{u}$ and we write $Q$ for the sum $\sum_{1}^{n} Q^{i}$ :

$$
\begin{aligned}
\varphi_{u}(x)=_{\text {def }} \sum_{i=1}^{n} u_{i}(x) & =\frac{1}{2} \sum_{1}^{n} x^{\prime} Q^{i} x+\sum_{1}^{n} b_{i}^{\prime} x \\
& =\frac{1}{2} x^{\prime}\left(\sum_{1}^{n} Q^{i}\right) x+\left(\sum_{1}^{n} b_{i}^{\prime}\right) x \\
& =\frac{1}{2} x^{\prime} Q x+\left(\sum_{1}^{n} b_{i}^{\prime}\right) x,
\end{aligned}
$$

and therefore

$$
\nabla \varphi_{u}(x)=\sum_{1}^{n} Q^{i} x+\sum_{1}^{n} b_{i}=Q x+\sum_{1}^{n} b_{i}
$$

and the matrix of second partials of $\varphi_{u}$ is simply $Q$.

The main objects of study will be the affective spaces. We assume that an $n$-tuple $\left(A_{i}\right)_{i \in N}$ is given, where for each $i \in N$ the affective space $A_{i}$ is a subspace of $\mathbb{R}^{m}$. The $n$-tuple $\left(A_{i}\right)_{1}^{n}$ is called a structure of affective spaces. The othogonal complement ${ }^{1}$ of each space $A_{i}$ is denoted $I_{i}$ and is called $i$ 's indifference space. A utility function $u_{i}$ is admissible for $i$ if it is constant with respect to $I_{i}$ and strictly convex with respect to $A_{i}$. (We consider convex functions instead of concave ones - i.e. we seek to minimize the aggregate objective function - in order to avoid the secondorder sign conditions that would make everything a bit messier.) Formally, the admissible functions for the space $A_{i}$ are the real-valued functions $u_{i}$ on $\mathbb{R}^{m}$ that satisfy the two conditions:

$$
u_{i}(x+z)=u_{i}(x), \quad \text { for all } x \in \mathbb{R}^{m} \text { and all } z \in I_{i},
$$

\footnotetext{
${ }^{1}$ We take $R^{m}$ to be Euclidean space, so that orthogonality of two vectors means that their scalar product (in the usual basis) is zero.
} 


$$
u_{i}((1-\lambda) x+\lambda y)<(1-\lambda) u_{i}(x)+\lambda u_{i}(y), \quad \text { if } x-y \notin I_{i} \text { and } 0<\lambda<1 .
$$

The quadratic $A_{i}$-admissible functions $u_{i}$ can be equivalently expressed in terms of the vectors $b_{i}$ and the matrices $Q^{i}$ that are admissible in the expression (1.1) for $u_{i}$ : the admissible vectors $b_{i}$ are precisely the members of $A_{i}$; and the admissible matrices $Q^{i}$ are the ones that satisfy the conditions in the following definition.

Definition 1.1. For a given subspace $A$ of $\mathbb{R}^{m}$, the $m \times m$ matrix $Q$ is admissible for $\boldsymbol{A}$, or $\boldsymbol{A}$-admissible, if it is symmetric and satisfies the following two conditions:

$A$ is the column space of $Q$

(i.e. the space spanned by the columns of $Q$ ),

$x^{\prime} Q x>0$, for all $x \in A \backslash\{0\}$.

A profile $\left(Q^{1}, \ldots, Q^{n}\right)$ of matrices is admissible for the structure $\left(A_{i}\right)_{1}^{n}$ of subspaces - we say the profile is $\left(\boldsymbol{A}_{i}\right)_{1}^{n}$-admissible - if each component $Q^{i}$ is $A_{i}$-admissible.

We will assume throughout that the spaces $A_{i}$ taken together span $\mathbb{R}^{m}$. (There is no loss of generality here, for we could instead focus attention only on the subspace of $\mathbb{R}^{m}$ spanned by the sets $A_{i}$ and redefine that subspace as $\mathbb{R}^{m}$.) Thus, the matrix $Q$ will always be non-singular and there will be a unique program $x=\pi(u)$ that maximizes $\varphi_{u}$ : it is the solution of the equation

$$
Q x+\sum_{1}^{n} b_{i}=0 .
$$

We want to study the dependence of the solution $x$ upon variations in the vectors $b_{i}$, so we are interested in solutions of equations of the form:

$$
Q \Delta x+\Delta b_{i}=0 .
$$

The question we are interested in, as described in the Introduction, is whether, for a given pair of agents $j$ and $k$, there is an admissible change in agent $j$ 's utility function for which the resulting change in $x$ will affect $k$ 's utility. In other words, is there a vector $\Delta b_{j}$ (which must be drawn from the space $A_{j}$ ) for which the solution $\Delta x$ does not lie in $I_{k}$ ? The existence of such a vector is equivalent to the statement that $A_{j}$ is not a subset of $Q\left(I_{k}\right)$. We will say that $\boldsymbol{j}$ cannot affect $\boldsymbol{k}$ if $A_{j} \subseteq Q\left(I_{k}\right)$ for every profile $Q=\left(Q^{1}, \ldots, Q^{n}\right)$ of admissible matrices. If $j$ can affect $k$ - if there is a $Q$ for which $A_{j} \nsubseteq Q\left(I_{k}\right)$ - then in fact every neighborhood in the space of admissible matrices will contain such a $Q$. Thus, the following definition is the one that we want to make.

Definition 1.2. $A_{j}$ can affect $A_{k}$ in $\left(A_{i}\right)_{1}^{n}$ if there is an $\left(A_{i}\right)_{1}^{n}$-admissible profile $\boldsymbol{Q}=\left(Q^{1}, \ldots, Q^{n}\right)$ for which $A_{j} \nsubseteq Q\left(I_{k}\right)$, where $Q=\sum_{1}^{n} Q^{i}$.

It will be shown in Section 4 that two agents can affect one another in a given 
structure if and only if they cannot be separated by a decomposition of the structure. Decomposition of structures will be defined and characterized in terms of matrices in Section 3, after we establish, in Section 2, how to characterize the admissible matrices in terms of alternative bases.

\section{Changing the basis}

In Section 1 the framework for our analysis was laid out entirely in terms of the usual basis for $\mathbb{R}^{m}{ }^{2}$ The analysis will depend heavily upon changes in the basis, however, and it will be important to have a notation that takes account of different bases. Let $\mathscr{E}$ denote the usual basis for $\mathbb{R}^{m}$, consisting of the $m$ unit vectors $e_{1}, \ldots, e_{m}$. Let $\mathscr{B}$ be an arbitrary basis for $\mathbb{R}^{m}$, consisting of the $m$ vectors $\beta_{1}, \ldots$, $\beta_{m}$, and let $C$ be the matrix whose rows are the members of $\mathscr{B}$, expressed in terms of $\mathscr{E}$. We will use the notation $\tilde{x}$ and $\tilde{Q}$ to represent the conversion of a vector $x$ and a matrix $Q$ from $\mathscr{E}$-coordinates to $\mathscr{B}$-coordinates, in the following sense (the notation $M^{\mathrm{t}}$ will always denote the transpose of the matrix $M$ ):

$$
\tilde{x}=C x \text { and } \tilde{Q}=\left(C^{-1}\right)^{t} Q C^{-1} \text {. }
$$

Thus, we have, for example,

Remark 2.1. $\tilde{x}^{\mathrm{t}} \tilde{Q} \tilde{x}=(C x)^{\mathrm{t}}\left(C^{\mathrm{t}}\right)^{-1} Q C^{-1}(C x)=x^{\mathrm{t}} C^{\mathrm{t}}\left(C^{\mathrm{t}}\right)^{-1} Q C^{-1} C x=x^{\mathrm{t}} Q x$.

We will also define a second coordinate tranformation in terms of the basis $\mathscr{B}$, using in this case the matrix $C^{\mathrm{t}}$ (whose columns are the members of $\mathscr{B}$ ):

$$
\hat{x}=\left(C^{\mathrm{t}}\right)^{-1} x \text {, i.e. } x=C^{\mathrm{t}} \hat{x} \text {. }
$$

A few additional elementary properties of these coordinate changes are given in the following remarks. Indeed, Remarks $2.1,2.3$, and 2.4 are the motivation for using these two particular transformations.

Remark 2.2. $\tilde{x}^{\mathrm{t}} \hat{y}=x^{\mathrm{t}} y$.

Remark 2.3. $y=Q x$ if and only $\hat{y}=\tilde{Q} \tilde{x}$.

Remark 2.4. If a subset $\left\{\beta_{k} \mid k \in K\right\}$ of $\mathscr{B}$ is a basis for a subspace $S$ of $\mathbb{R}^{m}$, then $x \in S$ if and only if $\hat{x}_{k}=0$ for every $k \notin K$, and $x \in S^{\perp}$ if and only if $\tilde{x}_{k}=0$ for every $k \in K$.

Definition 2.1. Given a basis $\mathscr{B}$ and a subspace $A$ in $\mathbb{R}^{m}$, an $m \times m$ matrix $Q$ is admissible for $A$ and $\mathscr{B}$, also written $A, \mathscr{B}$-admissible, if there is an $A, \mathscr{E}$-admissible

\footnotetext{
${ }^{2}$ Except for the definition of the indifference space $I_{t}$, the basis was irrelevant. It is already clear, however, that the relation between the sets $A_{t}$ and $I_{t}$ is the central feature of the theory, and that we therefore cannot ignore the basis that is being used.
} 
matrix $S$ (see Definition 1.2) for which $Q=\bar{S}$. A profile $\left(Q^{1}, \ldots, Q^{n}\right)$ of matrices is admissible with respect to $\mathscr{B}$ for the structure $\left(A_{i}\right)_{1}^{n}$ - we also say the profile is $\left(A_{i}\right)_{1}^{n}, \mathscr{B}$-admissible - if each component $Q^{i}$ is $A_{i}, \mathscr{B}$-admissible for $A_{i}$.

Example. If the first $k$ members of $\mathscr{B}$ constitute a basis for $A$, then the $A, \mathscr{B}$ admissible matrices are the ones for which the $k \times k$ principal submatrix at the upper left is positive definite and for which all entries not in that submatrix are zero.

The following two lemmas give properties of $A, \mathscr{B}$-admissible matrices that we will need later in the paper. Lemma 2.1 simply assures us that (1.3) and (1.4) can be used to describe admissibility no matter what the basis.

Lemma 2.1. $\tilde{Q}$ is $A, \mathscr{B}$-admissible if and only if

The column space of $\tilde{Q}$ is the set $A$, where members of $A$ are expressed in terms of the basis $\mathscr{B}$;

and

$\tilde{Q}$ is positive definite with respect to $A-i$.e. if $\tilde{x}$ is the expression in terms of $\mathscr{B}$ of a member $x \neq 0$ of $A$, then $\tilde{x}^{\mathrm{t}} \tilde{Q} \tilde{x}>0$.

Proof. (2.1) is simply a restatement of Remark 2.3, and (2.2) is a restatement of Remark 2.1.

Lemma 2.2. The set of all $A, \mathscr{B}$-admissible matrices is a convex cone.

Proof. Let $Q$ and $Q^{\prime}$ be $A, \mathscr{B}$-admissible and let $\lambda$ be a positive real number. We must show that

and

$$
Q+Q^{\prime} \text { is } A, \mathscr{B} \text {-admissible, }
$$

$\lambda Q$ is $A, 98$-admissible.

In light of Lemma $2.1,(2.4)$ is obvious. For (2.3), we note first that $x^{\mathrm{t}}\left(Q+Q^{\prime}\right) x=$ $x^{\mathrm{t}} Q x+x^{\mathrm{t}} Q^{\prime} x$, which ensures that $Q+Q^{\prime}$ is positive definite with respect to $A$. This also establishes that

$$
\operatorname{Rank}\left(Q+Q^{\prime}\right) \geq \operatorname{Rank} Q=\operatorname{Rank} Q^{\prime} .
$$

It is easy to see that the column space of $Q+Q^{\prime}$ is a subspace of $A$ (the column space of both $Q$ and $Q^{\prime}$ ), and this, together with (2.5), establishes that $A$ is the column space of $Q+Q^{\prime}$. Application of Lemma 2.1 yields (2.3).

A useful alternative characterization of the set of admissible matrices for a basis $\mathscr{B}$ and a subspace $A$ is as follows: a matrix is $A, \mathscr{B}$-admissible if and only if it can 
be expressed as the product $M^{\mathrm{t}} M$ of a matrix $M$ and its tranpose, where the rows of $M$ constitute a basis for $A$ (expressed in terms of the basis $\mathscr{B}$ for $\mathbb{R}^{m}$ ). This characterization and several of its useful properties are established in Appendix A.

\section{Decomposability and block-diagonality}

In this section we define and develop the concept of a decomposition of a structure $\left(A_{i}\right)_{i \in N}$ of affective spaces. As in the example in the Introduction, a decomposition of the structure $\left(A_{i}\right)_{i \in N}$ is a separation of the aggregate optimization problem into two or more independent optimization problems, the individual solutions of which provide a solution of the aggregate problem.

Definition. A decomposition of $\left(A_{i}\right)_{i \in N}$ is a pair $\left(P,\left(Z_{p}\right)_{p \in P}\right)$, where

(1) $P$ is a partition of $N$;

(2) For each $p \in P, Z_{p}$ is a subspace of $\mathbb{R}^{m}$ such that

(a) if $i \in p$, then $A_{i} \subseteq Z_{p}$,

(b) $Z_{p} \cap \sum_{q \neq p} Z_{q}=\{0\}$.

A decomposition is non-trivial if the partition $P$ is non-trivial (i.e. if $P$ is not a singleton). We will write $[i]_{P}$, or just [i], for the equivalence class of $i$ in $P$. Notice that when the sets $A_{i}$ taken together span $\mathbb{R}^{m}$, it follows a fortiori that the spaces $Z_{p}$ together span $\mathbb{R}^{m}$.

Definition. $\left(A_{i}\right)_{1}^{n}$ is decomposable if it has a non-trivial decomposition. Otherwise it is indecomposable.

The two theorems to be given in this section will characterize decomposability in terms of the matrices $Q=\sum_{1}^{n} Q^{i}$ - specifically, $\left(A_{i}\right)_{1}^{n}$ is shown to be decomposable if and only if there is a basis with respect to which every admissible matrix is blockdiagonal, with blocks corresponding to the decomposing spaces $Z_{k}$.

Theorem 3.1. Let $\left(\left\{N_{1}, \ldots, N_{K}\right\},\left(Z_{k}\right)_{1}^{K}\right)$ be a decomposition of $\left(A_{i}\right)_{1}^{n}$, where the spaces $A_{i}$ span $\mathbb{R}^{m}$, and let $\mathscr{B}$ be a basis for $\mathbb{R}^{m}$, ordered in such a way that the first $m_{1}$ members are a basis for $Z_{1}$, the next $m_{2}$ members a basis for $Z_{2}$, and so on. Then for every profile $\left(Q^{1}, \ldots, Q^{n}\right)$ that is admissible for $\left(A_{i}\right)_{1}^{n}$, the matrix $\tilde{Q}=$ $\sum_{1}^{n} \tilde{Q}^{i}$ has the block-diagonal form:

$$
\tilde{Q}=\left[\begin{array}{ccccc}
B_{1} & 0 & 0 & \ldots & 0 \\
0 & B_{2} & & & \vdots \\
0 & & \ddots & \vdots \\
\vdots & & & \vdots & \vdots \\
0 & \ldots & \ldots & B_{K}
\end{array}\right],
$$

where each $B_{k}$ is $m_{k} \times m_{k}$ and positive definite. 
Proof. Let $k \in\{1, \ldots, K\}$, let $i \in N_{k}$, and partition $\tilde{Q}^{i}$ into blocks $B_{r s}$ of dimension $m_{r} \times m_{s}$ :

$$
\tilde{Q}^{i}=\left[\begin{array}{ccc}
B_{11} & \ldots & B_{1 K} \\
\vdots & & \vdots \\
B_{K 1} & \ldots & B_{K K}
\end{array}\right]
$$

We will show that each block $B_{r s}$ is zero except when $r=s=k$. This will suffice, because $\tilde{Q}=\sum_{1}^{n} \tilde{Q}^{i}$. We have $Q^{i} z \in A_{i}$ for all $z \in \mathbb{R}^{m}$; a fortiori, $Q^{i} z \in Z_{k}$ for all $z \in \mathbb{R}^{m}$. In other words, if we write $Q^{i} z=y$, and we partition the vector $y$ into blocks, or 'segments', that correspond to the block-partitioning of $\tilde{Q}$ - i.e. $y=$ $\left(y_{1}, \ldots, y_{K}\right)$, where each segment $y_{j}$ contains $m_{j}$ components - then according to Remark 2.4 we have $\hat{y}_{j}=0$ for $j \neq k$. Thus, according to Remark 2.3 (and because $z$ was an arbitrary member of $\mathbb{R}^{m}$ ), we have $B_{r s}=0$ if $r \neq k$ (i.e. the only non-zero blocks are in the $k$ th row of blocks). Because $\tilde{Q}^{i}$ is symmetric, we must in fact have, as desired, $B_{r s}=0$ unless $r=s=k$. Positive definiteness of all the diagonal blocks $B_{k k}$ in $\tilde{Q}$ follows from the block-diagonality and positive definiteness of $\tilde{Q}$.

In Theorem 3.1 it was important to consider an ordering of the members of the basis for $\mathbb{R}^{m}$, so that we could partition matrices into blocks that correspond to the decomposition of $\left(A_{i}\right)_{1}^{n}$. This notion of an 'ordered' basis will continue to be important. Since the only sets to be endowed with an order will be finite sets, an ordered set will sometimes be more conveniently written as a $\xi$-tuple (no two of whose components are equal), where $\xi$ is a positive integer. Intervals, or 'segments', of ordered sets will also be important:

Definition. A segment of a $\xi$-tuple $\left(x_{1}, \ldots, x_{\xi}\right)$ is a $k$-tuple $\left(x_{r+1}, \ldots, x_{r+k}\right)$, where $r+1 \geq 1$ and $r+k \leq \xi$.

Thus, in the statement of Theorem $3.1, \mathscr{B}$ is an ordered basis for $\mathbb{R}^{m}$, consisting of $K$ non-overlapping segments, in which (for each $k$ ) segment $k$ is an ordered basis for $Z_{k}$ (the order within the segments was irrelevant to the statement and proof).

Theorem 3.2. If there is an ordered basis $\mathscr{B}$ for $\mathbb{R}^{m}$, made up of segments $\mathscr{B}_{k}$ $(k=1, \ldots, K)$ of length $m_{k}$, and such that for every $\left(A_{i}\right)_{1}^{n}$-admissible profile $\left(Q^{1}, \ldots, Q^{n}\right)$ the matrix $\tilde{Q}=\sum_{1}^{n} \tilde{Q}^{i}$ has the form (3.1), then there is a decomposition $\left(\left\{N_{1}, \ldots, N_{K}\right\},\left(Z_{k}\right)_{1}^{K}\right)$ of $\left(A_{i}\right)_{1}^{n}$ in which, for each $k$, the segment $\mathscr{B}_{k}$ is a basis for $Z_{k}$.

Proof. Let $\left(Q^{1}, \ldots, Q^{n}\right)$ be an arbitrary $\left(A_{i}\right)_{1}^{n}$-admissible profile of matrices, and let $i$ be an arbitrary agent in $N$. We will show that

(1) $\bar{Q}^{l}$ has the block-diagonal form (3.1), and

(2) all but one of the diagonal blocks in $\tilde{Q}^{i}$ are zero.

Then we will use (1) and (2) to show that $A_{i} \subseteq Z_{k}$ for all $i \in N_{k}$. This will suffice, 
because then we simply define each set $N_{k}$ as the set of agents $i$ for whom the $k$ th diagonal block is non-zero.

(1) According to the theorem's assumption, $\tilde{Q}$ has the form (3.1). Suppose an off-diagonal block $B_{r s}$ of $\tilde{Q}^{i}$ is not zero. Then we can perturb the matrix $Q^{i}$ in an admissiblc way (c.g. multiply it by $1+\delta$ ), with the result that $\tilde{Q}$ (which is the sum of the $\tilde{Q}^{i}$ ) will no longer have the form (3.1), because its corresponding offdiagonal block will no longer be zero.

(2) Suppose that two of the diagonal blocks of $\tilde{Q}^{i}$, say $B_{r r}$ and $B_{s s}(r \neq s)$, are not zero. Then, according to Lemmas A.2 and A.3 in Appendix A, there is an $A_{i^{-}}$ admissible matrix in which the $B_{r s}$ block is not zero, contradicting what we have just established in (1).

Now let $y \in A_{i}$; we must show that $y \in Z_{k}$. Since $A_{i}$ is the column space of $Q^{i}$, there is a vector $x \in \mathbb{R}^{m}$ for which $Q^{i} x=y$-i.e. $\tilde{Q}^{i} \tilde{x}=\hat{y}$. But it is clear from the form that we have just established for $\tilde{Q}^{i}$ that if we partition $\hat{y}$ into segments with the same lengths as the segments $\mathscr{B}_{k}$ of $\mathscr{B}$, then the only non-zero segment of $\hat{y}$ will be the $k$ th one - in other words, according to Remark 2.4, $y \in Z_{k}$, as desired.

The results in this section can be summarized as follows: the structure $\left(A_{i}\right)_{1}^{n}$ of spaces is decomposable if and only if, in some basis, every admissible aggregate matrix $Q=\sum_{1}^{n} Q^{i}$ is block-diagonal.

\section{Decomposability and affectability}

The two theorems to be given in this section constitute the paper's main result, namely that two agents can affect one another in a given structure of spaces if and only if they cannot be separated - i.e. if and only if the structure cannot be decomposed in such a way that the two agents are in separate component parts of the decomposition.

Definition. Two agents $j$ and $k$ are separable in $\left(A_{i}\right)_{i \in N}$ if there is a decomposition $\left(P,\left(Z_{p}\right)\right)$ of $\left(A_{i}\right)_{i \in N}$ in which $[j] \neq[k]-$ i.e. in which $j$ and $k$ are in distinct members of $P$.

Theorem 4.1. If $A_{j}$ and $A_{k}$ are separable in $\left(A_{i}\right)_{1}^{n}$, then $A_{j}$ and $A_{k}$ cannot affect one another.

Proof. Let $\left(\left\{N_{j}, N_{k}\right\},\left(Z_{j}, Z_{k}\right)\right)$ be a decomposition of $\left(A_{i}\right)_{1}^{n}$ in which $j \in N_{j}$ and $k \in N_{k}$. Let $\mathscr{B}_{j}$ and $\mathscr{B}_{k}$ be bases of $Z_{j}$ and $Z_{k}$, and let $\mathscr{B}=\mathscr{B}_{j} \cup \mathscr{B}_{k}$, ordered so that the members of $\mathscr{B}_{j}$ come first. Then Theorem 3.1 guarentees that for every admissible profile $\left(Q^{1}, \ldots, Q^{n}\right)$ the matrix $\tilde{Q}=\sum_{1}^{n} \tilde{Q}^{i}$ will have the form:

$$
\tilde{Q}=\left[\begin{array}{cc}
B_{j} & 0 \\
0 & B_{k}
\end{array}\right] .
$$


If we partition vectors of $\mathbb{R}^{m}$ in the same way - i.e. $y=\left(y_{j}, y_{k}\right)$ - then $y$ will be an element of $A_{j}$ or $A_{k}$ if and only if $\hat{y}_{k}=0$ or $\hat{y}_{j}=0$, respectively. Let $y \in A_{j}$ and let $z=Q^{-1} y$; then we have:

$$
\tilde{z}=\tilde{Q}^{-1} \hat{y}=\left[\begin{array}{c}
B_{j}^{-1} y \\
B_{k}^{-1} y
\end{array}\right]=\left[\begin{array}{c}
B_{j}^{-1} y \\
0
\end{array}\right] .
$$

Thus, according to Remark $2.4, z \in I_{i}$, as desired.

The converse of Theorem 4.1 is Theorem 4.2 , below, which is not nearly so easy to prove. The difficult part of the proof will be isolated as Lemma 4.2, below. The proofs of Lemmas 4.1 and 4.2 will both be deferred until after they have been used to prove Theorem 4.2 and until after some additional ideas havc been developed that will help us to prove the two lemmas.

The first of the two lemmas tells us that if one agent can affect another, then adding an agent will not change that fact.

Lemma 4.1. If $A_{j}$ can affect $A_{k}$ in $\left(A_{i}\right)_{1}^{n}$, then $A_{j}$ can affect $A_{k}$ in $\left(A_{i}\right)_{1}^{n+1}$.

The second lemma tells us that if two agents are separable, then adding an agent will not change that fact unless the addition allows one of the agents to affect the other.

Lemma 4.2. If $A_{j}$ and $A_{k}$ are separable in $\left(A_{i}\right)_{1}^{n}$, and if $A_{k}$ cannot affect $A_{j}$ in $\left(A_{i}\right)_{1}^{n+1}$, then $A_{j}$ and $A_{k}$ are separable in $\left(A_{i}\right)_{1}^{n+1}$.

With the two lemmas in hand, Theorem 4.2 is easy.

Theorem 4.2. If $A_{j}$ cannot affect $A_{k}$ in $\left(A_{i}\right)_{1}^{n}$, then $A_{j}$ and $A_{k}$ are separable in $\left(A_{i}\right)_{1}^{n}$.

Proof. Without loss of generality, let $j=1$ and $k=2$. Lemma 4.1, applied recursively, ensures that $A_{2}$ cannot affect $A_{1}$ in $\left(A_{i}\right)_{1}^{K}$ for $K=n-1, n-2, \ldots, 3,2$. In the case $K=2$ it is easy to show that, as a consequence, $A_{1}$ and $A_{2}$ are separable in $\left(A_{i}\right)_{1}^{2}$. Now we can apply Lemma 4.2 recursively to establish that $A_{1}$ and $A_{2}$ are separable in $\left(A_{i}\right)_{1}^{K}$ for $K=3, \ldots, n$.

Now we must turn to proving Lemmas 4.1 and 4.2. We first derive from the "cannot affect' relation, $A_{2} \cong Q\left(I_{1}\right)$, a rank condition on the matrix $\tilde{Q}$.

Lemma 4.3. Let $A_{1} \cap A_{2}=\{0\}$ and let $\left(Q^{1}, \ldots, Q^{n}\right)$ be an $\left(A_{i}\right)_{1}^{n}, \mathscr{E}$-admissible profile of $n$ rices. Let $\mathscr{B}_{1}$ and $\mathscr{B}_{2}$ be ordered bases for $A_{1}$ and $A_{2}$; let $\mathscr{B}_{3}$ be an ordered basis for $\left(A_{1}+A_{2}\right)^{\perp}$; and let $\mathscr{B}$ be the ordered basis made up of the segments $\mathscr{B}_{1}, \mathscr{B}_{2}$, and $\mathscr{B}_{3}$, in that order. Let $\tilde{Q}$ be partitioned into blocks cor- 
responding to the segments of $\mathscr{B}$, as follows:

$$
\tilde{Q}=\left[\begin{array}{lll}
B_{11} & B_{12} & B_{13} \\
B_{21} & B_{22} & B_{23} \\
B_{31} & B_{32} & B_{33}
\end{array}\right],
$$

and let $\bar{B}$ be the block matrix formed by deleting from $\tilde{Q}$ the blocks in row 2 and column 1:

$$
\bar{B}=\left[\begin{array}{ll}
B_{12} & B_{13} \\
B_{32} & B_{33}
\end{array}\right] .
$$

If $A_{2}$ cannot affect $A_{1}$, then $\operatorname{Rank}(\bar{B})=\operatorname{Rank}\left(B_{33}\right)$ - i.e. adding the specified borders to $B_{33}$ does not increase its rank.

Proof. Assume that $A_{2}$ cannot affect $A_{1}$ - i.e $A_{2} \cong Q\left(I_{1}\right)$ - which is equivalent to the proposition that for every $y \in \mathbb{R}^{m}$,

$$
y \in A_{2} \Rightarrow \exists z \in \mathbb{R}^{m}:\left(z \in I_{1} \& Q z=y\right) .
$$

Partitioning $m$-vectors $x$ into segments $x_{(1)}, x_{(2)}$, and $x_{(3)}$ that correspond to the partitioning of $\tilde{Q}$, and applying Remarks 2.3 and 2.4, we see that (4.3) is equivalent to

$$
\left[\hat{y}_{(1)}=0 \& \hat{y}_{(3)}=0\right] \Rightarrow \exists z \in \mathbb{R}^{m}:\left[\tilde{z}_{(1)}=0 \& \tilde{Q} \tilde{z}=\hat{y}\right],
$$

which (discarding the ${ }^{\wedge}$ and ${ }^{\sim}$ notations) is in turn equivalent to the statement that for every $y_{(2)} \in \mathbb{R}^{m}$, there is a solution $\left(z_{(2)}, z_{(3)}\right)$ of the following system:

$$
\begin{aligned}
& B_{12} z_{(2)}+B_{13} z_{(3)}=0, \\
& B_{22} z_{(2)}+B_{23} z_{(3)}=y_{(2)}, \\
& B_{32} z_{(2)}+B_{33} z_{(3)}=0 .
\end{aligned}
$$

It therefore follows that the dimension of the set of solutions to the system,

$$
\left[\begin{array}{ll}
B_{12} & B_{13} \\
B_{32} & B_{33}
\end{array}\right]\left[\begin{array}{l}
z_{(2)} \\
z_{(3)}
\end{array}\right]=\left[\begin{array}{l}
0 \\
0
\end{array}\right] \text {, }
$$

is at least as large as the dimension of $A_{2}$.

Let $m_{1}, m_{2}$, and $m_{3}$ denote the dimensions of $A_{1}, A_{2}$, and $\left(A_{1}+A_{2}\right)^{\perp}$; let $\sigma$ denote the dimension of the set of solutions to (4.5); and let $\varrho$ denote the rank of the matrix $\bar{B}$. Then $\sigma+\varrho=m_{2}+m_{3}$. But we have just shown that $\sigma \geq m_{2}$; thus, $\varrho \leq m_{3}$. Moreover, $\operatorname{Rank}\left(B_{33}\right) \leq \varrho$, by definition. But $B_{33}$ is non-singular (because $\tilde{Q}$ is positive definite) - i.e. $\operatorname{Rank}\left(B_{33}\right)=m_{3}$ - which yields the desired conclusion that $\varrho=\operatorname{Rank}\left(B_{33}\right)$.

Now we are prepared to prove Lemma 4.1.

Proof of Lemma 4.1. Assume that $A_{1}$ cannot affect $A_{2}$ in $\left(A_{i}\right)_{1}^{n+1}$; we must show 
that $A_{1}$ also cannot affect $A_{2}$ in $\left(A_{i}\right)_{1}^{n}$. If $A_{1} \cap A_{2} \neq\{0\}$, then it is easy to show that $A_{1}$ can affect $A_{2}$ in any structure $\left(A_{i}\right)$ of spaces that includes them; we therefore have $A_{1} \cap A_{2}=\{0\}$.

Let $\left(Q^{1}, \ldots, Q^{n}\right)$ be an $\left(A_{i}\right)_{1}^{n}$-admissible profile of matrices; let $Q=\Sigma_{1}^{n} Q^{i}$; and let $Q^{\prime}$ be an $A_{n+1}$-admissible matrix. Because $A_{1} \cap A_{2}=\{0\}$, we can partition both $\tilde{Q}$ and $\tilde{Q}^{\prime}$ as in (4.1); let $B_{r s}$ and $B_{r s}^{\prime}$ denote the blocks of $\tilde{Q}$ and $\tilde{Q}^{\prime}$.

We are going to consider matrices $\tilde{Q}+\lambda \tilde{Q}^{\prime}$ for positive real numbers $\lambda$. Because $A_{1}$ cannot affect $A_{2}$ in $\left(A_{i}\right)_{1}^{n+1}$, we can apply Lemma 4.3 to these matrices. Specifically, we know that any column drawn from the left segment of $\bar{B}$ in (4.2) must be a linear combination of the columns in the right segment: let $b$ and $b^{\prime}$ be (arbitrary) corresponding columns in the matrices

$$
\left[\begin{array}{l}
B_{12} \\
B_{32}
\end{array}\right] \text { and }\left[\begin{array}{l}
B_{12}^{\prime} \\
B_{32}^{\prime}
\end{array}\right]
$$

then for every $\lambda>0$ there is a vector $x \in \mathbb{R}^{m}$ that satisfies

$$
b+\lambda b^{\prime}=\left[\left[\begin{array}{l}
B_{13} \\
B_{33}
\end{array}\right]+\lambda\left[\begin{array}{l}
B_{13}^{\prime} \\
B_{33}^{\prime}
\end{array}\right]\right] x .
$$

We must show that (4.6) is true for $\lambda=0$ as well. (Note that this is indeed sufficient, because $B_{33}$ is non-singular, which implies that augmenting $B_{33}$ with $B_{13}$ does not increase its rank.)

Because $B_{33}+\lambda B_{33}^{\prime}$ is non-singular, (4.6) is true (for each positive $\lambda$ ) for the unique $x(\lambda)$ defined by

$$
x(\lambda)=\left(B_{33}+\lambda B_{33}^{\prime}\right)^{-1}\left(b+\lambda b^{\prime}\right) .
$$

If we also define $x(0)$ by equation (4.7) - i.e. $x(0)=B_{33}^{-1} b$ - then (4.6) holds true for $\lambda=0$ : the limit (as $\lambda$ decreases to 0 ) of each term involving $\lambda$ is equal to its value at $\lambda=0$.

For Lemma 4.2 we must partition matrices $Q$ into blocks in the same way that we have already been doing in this section - namely, in a way that keeps agents 1 and 2 separate from the other agents - but we must also distinguish among those other agents: we must keep track of which of them are in the class [1], which are in the class [2], and which are in neither class and therefore separable from 1 and 2. We will use the following notation and terminology:

If $A_{1}$ and $A_{2}$ are separable in $\left(A_{i}\right)_{1}^{n}$, let $\left(N_{1}, N_{2}, N_{3} ;\left(Z_{1}, Z_{2}\right.\right.$, $\left.Z_{3}\right)$ ) be a decomposition of $\left(A_{i}\right)_{1}^{n}$ in which $N_{1}=$ [1] and $N_{2}=$ [2] and in which $N_{1}$ and $N_{2}$ are minimal - that is, in which, for $j=1$ and $j=2, i \in N_{j}$ implies that $i$ and $j$ are not separable and in which the spaces $Z_{1}$ and $Z_{2}$ are also minimal, in the sense that for $j=1$ and $j=2, Z_{j}=\sum_{i \in[j]} A_{i}$. Partition $N_{1}$ and $N_{2}$ as follows: $N_{1^{\prime}}=\{1\} ; N_{2^{\prime}}=\{2\} ; N_{1^{n}}=[1] \backslash\{1\} ; N_{2^{n}}=$ $[2] \backslash\{2\}$. A well-ordered basis for $\mathbb{R}^{m}$ is an ordered basis $\mathscr{B}$ 
composed of the segments (in this order) $\mathscr{B}_{1^{\prime}}, \mathscr{B}_{2^{\prime}}, \mathscr{B}_{1^{*}}, \mathscr{B}_{2^{n}}$, $\mathscr{B}_{3}$, where $\mathscr{B}_{1^{\prime}}$ and $\mathscr{B}_{2^{\prime}}$ are ordered bases for $A_{1}$ and $A_{2}$; $\mathscr{B}_{1^{\prime}} \cup \mathscr{B}_{1^{\prime \prime}}$ and $\mathscr{B}_{2^{\prime}} \cup \mathscr{B}_{2^{\prime \prime}}$ are ordered bases for $Z_{1}$ and $Z_{2}$; and $\mathscr{B}_{3}$ is an ordered basis for $Z_{3}$. Let $K=\left\{1^{\prime}, 2^{\prime}, 1^{\prime \prime}, 2^{\prime \prime}, 3\right\}$, and let $K$ be endowed with the order $1^{\prime}<2^{\prime}<1^{\prime \prime}<2^{\prime \prime}<3$. For each $k \in K$, let $m_{k}$ be the number of members of $\mathscr{B}_{k}$; and let $J=\{1, \ldots ; m\}$, with segments $J_{k}$ of length $m_{k}$. We will partition matrices $Q$ into blocks corresponding to the ordered set $K$ :

$$
Q=\left[\begin{array}{lllll}
B_{1^{\prime} 1^{\prime}} & B_{1^{\prime} 2^{\prime}} & B_{1^{\prime} 1^{\prime \prime}} & B_{1^{\prime} 2^{\prime \prime}} & B_{1^{\prime} 3} \\
B_{2^{\prime} 1^{\prime}} & B_{2^{\prime} 2^{\prime}} & B_{2^{\prime} 1^{\prime \prime}} & B_{2^{\prime} 2^{\prime \prime}} & B_{2^{\prime} 3} \\
B_{1^{\prime \prime} 1^{\prime}} & B_{1^{\prime \prime} 2^{\prime}} & B_{1^{\prime \prime} 1^{\prime \prime}} & B_{1^{\prime \prime} 2^{\prime \prime}} & B_{1^{\prime \prime} 3} \\
B_{2^{\prime \prime} 1^{\prime}} & B_{2^{\prime \prime} 2^{\prime}} & B_{2^{\prime \prime} 1^{\prime \prime}} & B_{2^{\prime \prime} 2^{\prime \prime}} & B_{2^{\prime \prime} 3} \\
B_{31^{\prime}} & B_{32^{\prime}} & B_{31^{\prime \prime}} & B_{32^{\prime \prime}} & B_{33}
\end{array}\right] .
$$

The following lemma says that if $A_{1}$ and $A_{2}$ are separable, then there is a wellordered basis in terms of which (a) changing any of the diagonal entries of an admissible matrix results in another admissible matrix, and (b) there is an admissible matrix in which every one of the 1 "- and $2^{\prime \prime}$-columns has a non-zero entry above the diagonal.

Lemma 4.4. If $A_{1}$ and $A_{2}$ are separable in $\left(A_{i}\right)_{1}^{n}$ then there is a well-ordered basis $\mathscr{B}$ that satisfies the following two conditions:

For each $k \in J$ : if $Q$ is $\left(A_{i}\right)_{1}^{n}$-admissible for $\mathscr{B}$, and if $Q^{\prime}$ satisfies both

(1) $Q_{k k}^{\prime}>Q_{k k}$ and

(2) $Q_{r s}^{\prime}=Q_{r s}$ if $r \neq k$ or $s \neq k$,

then $Q^{\prime}$ is also $\left(A_{i}\right)_{1}^{n}$-admissible for $\mathscr{B}$.

There is an $\left(A_{i}\right)_{1}^{n}, \mathscr{B}$-admissible matrix $Q$ in which, in every one of the $1^{\prime \prime}$ - and $2^{\prime \prime}$-columns, there is a non-zero entry above the diagonal - i.e. for every $k \in J_{1^{*}} \cup J_{2^{*}}$, there is $a j<k$ such that $Q_{j k}>0$.

Proof. We construct an appropriate basis $\mathscr{B}$ as follows. First, we construct a wellordered basis $\mathscr{B}$ that satisfies (4.10), and then we rearrange the order of $\mathscr{B}$ in such a way that (4.11) is satisfied, without disturbing the well-orderedness of $\mathscr{B}$.

Begin with the set $\mathscr{B}^{0}=\varnothing$. We engage in an $n$-step process in which, at each step $t$, we consider, or 'take', an agent $i(t)$ who has not been taken at any previous step, and we use that agent's space $A_{i(t)}$ to add some vectors to the ordered set $\mathscr{B}^{t-1}$, thereby obtaining an ordered set $\mathscr{B}^{t}$. The order in which we take the agents is simply any order consistent with the ordered set $K$ : for each $k \in K$, we take the members of $N_{k}$ in any order at all, but we consider the sets $N_{k}$ in the order $k=1^{\prime}, 2^{\prime}, 1^{\prime \prime}, 2^{\prime \prime}, 3$. At each step $t$, we add to $\mathscr{B}^{t-1}$ any vectors from $A_{i(t)}$ that will 
turn $\mathscr{B}^{t-1}$ into a basis $\mathscr{B}^{t}$ for the space $\Sigma_{\tau=1}^{t} A_{i(\tau)}$ - i.e. we add any set of linearly independent vectors in $A_{i(t)}$ that are not expressible in terms of the vectors in $\mathscr{B}^{t-1}$.

Let $\mathscr{B}$ denote the ordered set obtained at the end of the last step of the construction described in the preceding paragraph - i.e. $\mathscr{B}$ is the ordered set $\mathscr{B}^{n}$, endowed with the order in which its members were added. It is clear that $\mathscr{B}$ is a well-ordered basis. In order to show that it satisfies (4.10), let $k$ be an arbitrary member of $J$, let $t$ be the step at which the $k$ th member of $\mathscr{B}$ (say, $\beta_{k}$ ) was added, and let $i$ be the agent $i(t)$ from whose $A_{i}$ space the vector $\beta_{k}$ was drawn. Then the unit vector $e_{k}$ is the $\mathscr{B}$-representation of the vector $\beta_{k}$; if we add any positive multiple of $e_{k}$ to column $Q^{i}{ }_{k}$ of an $A_{i}, \mathscr{B}$-admissible matrix $Q^{i}$, the resulting matrix will still be $A_{i}, \mathscr{B}$-admissible (this is because the new matrix will continue to be symmetric and positive definite on $A_{i}$, since the only change is an increase in a diagonal entry; thus the rank will still be equal to the dimension of $A_{i}$; and therefore, since the new column space will clearly be a subspace of $A_{i}$, it will in fact be equal to $A_{i}$ ). We have shown, in other words, that for every component $k$, one of the matrices $Q^{i}$ can have its diagonal entry $Q_{k k}^{i}$ increased arbitrarily without destroying its admissibility; this is clearly sufficient to yield (4.10).

Now we must rearrange the order of $\mathscr{B}$ to achieve (4.11). We will first rearrange the order of the vectors in $\mathscr{R}_{1^{\prime \prime}}$. I .et $\xi=m_{1^{\prime}}+m_{2^{\prime}}$. Then $J_{1^{\prime \prime}}=\left\{\xi+1, \xi+2, \therefore, \xi+m_{1^{\prime \prime}}\right\}$ - in other words, $\mathscr{B}_{1^{\prime \prime}}$ consists of the vectors $\beta_{k}$ for which $k=\xi+1, \xi+2, \ldots$, $\xi+m_{1^{\prime \prime}}$. The rearrangement will be performed in a series of $m_{1^{\prime \prime}}$ steps, as follows.

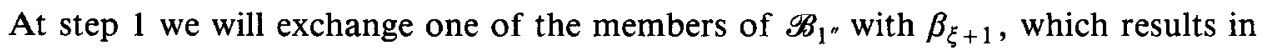

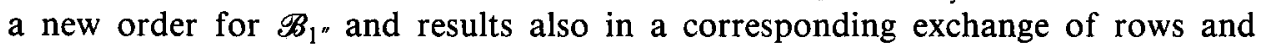
columns of the matrix $Q$. At step 2 we will exchange one of the remaining members of $\mathscr{B}_{1^{\prime \prime}}$ (i.e. one of the $\beta_{k}$ for $k=\xi+2, \ldots, \xi+m_{1^{\prime \prime}}$ ) with $\beta_{\xi+2}$, again giving us a new order for $\mathscr{B}_{1^{\prime \prime}}$ and a rearrangement of the matrix $Q$. We continue for a total of $m_{1^{\prime \prime}}$ steps, at each step $t$ exchanging $\beta_{\xi+t}$ with a later member of $\mathscr{B}_{1}$ " (under the order prevailing at that step), say $\beta_{k}$, where $\xi+t \leq k \leq \xi+m_{1^{\prime \prime}}$, thereby causing a corresponding exchange of row and column $\xi+t$ of matrix $Q$ with row and column $k$, and also rearranging the matrix $Q$ accordingly. It remains only to specify which vector $\beta_{k}$ (with $k \geq \xi+t$ ) will be exchanged with $\beta_{t}$ at step $t$. (Note that we allow at each step for the possibility of exchanging a vector with itself - i.e. for the null rearrangement.)

At each step $t$ of the rearranging process, suppose that for $s=\xi+t, \ldots, \xi+m_{1^{\prime \prime}}$, each of the columns $Q . s$ of every admissible matrix $Q$ has nothing but zero entries in the rows above row $\xi+t$ - i.e. $Q_{r s}=0$, when $r$ and $s$ satisfy both $r<\xi+t$ and $\xi+t \leq s \leq \xi+m_{1 "}$. Then Theorem 3.2 ensures that, contrary to assumption, $\mathscr{B}$ is not well-ordered, because either the set $N_{1}$ " is not minimal or else the space $Z_{1^{\prime \prime}}$ is not equal to $\sum_{i \in[1]} A_{i}$. Thus, at least one of these columns of at least one matrix $Q$ has a non-zero entry above row $\xi+t$; let that column be the one that is exchanged with column $\xi+t$, and exchange the same pair of rows in $Q$, and exchange the corresponding pair of vectors in $\mathscr{B}$. Clearly, in the new matrix $Q$, the column $\xi+t$ has 
a non-zero entry above the diagonal entry $Q_{\xi+t, \xi+t}$. Because the set of $\left(A_{i}\right)_{1}^{n}, \mathscr{B}$ admissible profiles is convex, there is in fact a matrix $Q$ that has a non-zero entry above every diagonal entry in $B_{1^{\prime \prime}} 1^{\prime \prime}$, as (4.11) requires.

Finally, we rearrange the segment $\mathscr{B}_{2^{\prime \prime}}$ of $\mathscr{B}$ in exactly the same way that we rearranged $\mathscr{B}_{1^{\prime \prime}}$, and the proof is complete.

We are finally prepared to give a proof of Lemma 4.2.

Proof of Lemma 4.2. Let $\mathscr{B}$ be a well-ordered basis that satisfies both (4.10) and (4.11) in $\left(A_{i}\right)_{1}^{n}$. Admissibility of matrices will be defined throughout in terms of the basis $\mathscr{B}$. Because $A_{1}$ and $A_{2}$ are separable in $\left(A_{i}\right)_{1}^{n}$, Theorem 3.1 guarantees that for every $\left(A_{i}\right)_{1}^{n}$-admissible profile $\left(Q^{1}, \ldots, Q^{n}\right)$ the matrix $Q=\sum_{1}^{n} Q^{i}$, which we will denote by $Q(n)$, must have the form:

$$
Q(n)=\left[\begin{array}{ccccc}
B_{1^{\prime} 1^{\prime}} & 0 & B_{1^{\prime} 1^{\prime \prime}} & 0 & 0 \\
0 & B_{2^{\prime} 2^{\prime}} & 0 & B_{2^{\prime} 2^{\prime \prime}} & 0 \\
B_{1^{\prime \prime} 1^{\prime}} & 0 & B_{1^{\prime \prime} 1^{\prime \prime}} & 0 & 0 \\
0 & B_{2^{\prime \prime} 2^{\prime}} & 0 & B_{2^{\prime \prime} 2^{\prime \prime}} & 0 \\
0 & 0 & 0 & 0 & B_{33}
\end{array}\right] .
$$

Still using the basis $\mathscr{B}$, we make use of Theorem 3.2 as follows. We show that, because $A_{2}$ cannot affect $A_{1}$ in $\left(A_{i}\right)_{1}^{n+1}$, matrices $Q=\Sigma_{1}^{N+1} Q^{i}$, which we will denote by $Q(n+1)$, must either have the form:

$$
Q(n+1)=\left[\begin{array}{ccccc}
B_{1^{\prime} 1^{\prime}} & 0 & B_{1^{\prime} 1^{\prime \prime}} & 0 & 0 \\
0 & B_{2^{\prime} 2^{\prime}} & 0 & B_{2^{\prime} 2^{\prime \prime}} & B_{2^{\prime} 3} \\
B_{1^{\prime \prime} 1^{\prime}} & 0 & B_{1^{\prime \prime} 1^{\prime \prime}} & 0 & 0 \\
0 & B_{2^{\prime \prime} 2^{\prime}} & 0 & B_{2^{\prime \prime} 2^{\prime \prime}} & B_{2^{\prime \prime} 3} \\
0 & B_{32^{\prime}} & 0 & B_{32^{\prime \prime}} & B_{33}
\end{array}\right],
$$

for every admissible profile $\left(Q^{1}, \ldots, Q^{n+1}\right)$, or else have the form:

$$
Q(n+1)=\left[\begin{array}{ccccc}
B_{1^{\prime} 1^{\prime}} & 0 & B_{1^{\prime} 1^{\prime \prime}} & 0 & B_{1^{\prime} 3} \\
0 & B_{2^{\prime} 2^{\prime}} & 0 & B_{2^{\prime} 2^{\prime \prime}} & 0 \\
B_{1^{\prime \prime} 1^{\prime}} & 0 & B_{1^{\prime \prime} 1^{\prime \prime}} & 0 & B_{1^{\prime \prime} 3} \\
0 & B_{2^{\prime \prime} 2^{\prime}} & 0 & B_{2^{\prime \prime} 2^{\prime \prime}} & 0 \\
B_{31^{\prime}} & 0 & B_{31^{\prime \prime}} & 0 & B_{33}
\end{array}\right],
$$

for every admissible profile $\left(Q^{1}, \ldots, Q^{n+1}\right)$. Then Theorem 3.2 will ensure that $A_{1}$ and $A_{2}$ are separable in $\left(A_{i}\right)_{1}^{n+1}$.

We have made use of the lemma's assumplion that $A_{1}$ and $A_{2}$ are separable in $\left(A_{i}\right)_{1}^{n}$ : it was that assumption that enabled us to write $Q(n)$ in the form (4.12). We will make use of the assumption that $A_{2}$ cannot affect $A_{1}$ in $\left(A_{i}\right)_{1}^{n+1}$ by invoking it in order to apply Lemma 4.3. In order to apply Lemma 4.3 to $Q(n+1)$, we rewrite the block-partitioned submatrix $\bar{B}$ of $Q(n+1)$, as defined in (4.2), in terms of the finer partitioning we are using herc (and we highlight the top and left borders of 
blocks):

$$
\bar{B}=\left[\begin{array}{l|lll}
B_{1^{\prime} 2^{\prime}} & B_{1^{\prime} 1^{\prime \prime}} & B_{1^{\prime} 2^{\prime \prime}} & B_{1^{\prime} 3} \\
\hline B_{1^{\prime \prime} 2^{\prime}} & B_{1^{\prime \prime} 1^{\prime \prime}} & B_{1^{\prime \prime} 2^{\prime \prime}} & B_{1^{\prime \prime} 3} \\
B_{2^{\prime \prime} 2^{\prime}} & B_{2^{\prime \prime} 1^{\prime \prime}} & B_{2^{\prime \prime} 2^{\prime \prime}} & B_{2^{\prime \prime} 3} \\
B_{32^{\prime}} & B_{31^{\prime \prime}} & B_{32^{\prime \prime}} & B_{33}
\end{array}\right] .
$$

Let $\dot{B}$ denote the 3-block $\times$ 3-block submatrix at the lower right. Because $A_{2}$ cannot affect $A_{1}$ in $\left(A_{i}\right)_{1}^{n+1}$, Lemma 4.3 guarantees that $\bar{B}$ has the same rank as $\dot{B}$.

We must show that each of the blocks $B_{1^{\prime} 2^{\prime}}, B_{1^{\prime} 2^{\prime \prime}}, B_{1^{\prime \prime} 2^{\prime}}$, and $B_{1^{\prime \prime} 2^{\prime \prime}}$ consists only of zeroes, and that the same is true either of $B_{1^{\prime} 3}$ and $B_{1^{\prime \prime} 3}$ or of $B_{2^{\prime} 3}$ and $B_{2^{\prime \prime} 3}$; symmetry of $Q(n+1)$ will then ensure that it has either the form (4.13) or (4.14). Let $Q$. ${ }_{s}$ denote an arbitrary column in the left border of $\bar{B}$ (i.e. $s \in J_{2}$ ). Because the ranks of $\bar{B}$ and $\dot{B}$ are the same, the column $Q_{s}$ is a linear combination of the nonborder columns:

$$
Q_{\cdot s}=\sum_{j \in J_{1^{*}}} \lambda_{j} Q_{\cdot j}+\sum_{j \in J_{2^{*}}} \lambda_{j} Q_{\cdot j}+\sum_{j \in J_{3}} \lambda_{j} Q_{\cdot j}
$$

Moreover, since $\grave{B}$ is non-singular, this linear combination is unique. We will show that $\lambda_{j}=0$ for each $j \in J_{1^{\prime \prime}}$ - i.e. that $Q_{\text {.s }}$ is actually a (unique) linear combination of only the $2^{\prime \prime}$ - and 3 -columns of $\dot{B}$. We adopt the notational simplification $J^{*}=$ $J_{1^{\prime \prime}} \cup J_{2^{\prime \prime}} \cup J_{3}$; thus, (4.16) becomes $Q . s=\sum_{j \in J^{*}} \lambda_{j} Q_{. j}$.

We consider the $1^{\prime \prime}$-columns (i.e. the indices $j \in J_{1^{\prime \prime}}$ ) one at a time, moving from left to right. Thus, let $k$ be the first member of $J_{1^{\prime \prime}}$. Because $\mathscr{B}$ satisfies (4.11), there is a non-zero entry $q_{r k}$ where $r<j$. Moreover, the row $r$ can be chosen so that $r \notin J_{2^{\prime}}$. [It can certainly be so chosen in $Q(n)$; if that entry becomes zero in $Q(n+1)$ - i.e. when $Q^{n+1}$ is added to $Q(n)$ - then it can be made non-zero by replacing $\left(Q^{1}, \ldots, Q^{n}\right)$ with $\xi\left(Q^{1}, \ldots, Q^{n}\right)$ for arbitrarily small $\xi$.] Within the row $Q_{r}$, let $c$ denote the row vector consisting only of components $q_{r j}$ where $j \in J^{*}$ - i.e. components in the 1"-, 2"-, and 3-columns; clearly, the first component of $c$ is non-zero. Note that $q_{r s}=\sum_{j \in J^{*}} \lambda_{j} c_{j}=c \cdot \lambda$, according to (4.16). Because $\mathscr{B}$ satisfies (4.10), we can perturb the diagonal entries of $Q$ without destroying its admissibility. Thus, according to Lemmas B.2 and B.3 of Appendix B, if $\lambda_{k} \neq 0$, then there is an admissible matrix $Q^{\prime}$ in which the row $Q_{r}^{\prime}$. is the sante as the corresponding row $Q_{r}$. of $Q$, but for which the coefficients $\lambda_{j}^{\prime}$ that correspond to the $\lambda_{j}$ in (4.16) satisfy $c \cdot \lambda^{\prime} \neq$ $c \cdot \lambda$ - i.e. $q_{r s}^{\prime} \neq q_{r s}$, which cannot be true, because $Q_{r}^{\prime}=Q_{r}$. . The contradiction establishes that $\lambda_{k}$ must be zero.

Now we have established that each of the 2 '-columns is a (unique) linear combination of the columns $Q . j$ for $j \in J^{*} \backslash\{k\}$, so we can repeat the argument of the preceding paragraph, replacing $J^{*}$ with $J^{*} \backslash\{k\}$, thereby establishing that the next coefficient, $\lambda_{k+1}$, is also zero. Continuing in the same way, we eventually show that there is an admissible $Q$ in which $\lambda_{j}=0$ for every $j \in J_{1}^{\prime \prime}-$ i.e. each of the $2^{\prime}$ columns is a unique linear combination of just the $2^{\prime \prime}$ - and 3-columns. The same recursive argument also establishes that each of the $1^{\prime}$-rows in (4.15) is a unique 
linear combination of just the $1^{\prime \prime}$ - and 3-rows. [For each $k \in J_{2^{\prime \prime}}$ the non-zero abovethe-diagonal entry could lie in the block $B_{2^{\prime} 2^{\prime \prime}}$, which is not in the matrix $\bar{B}$; symmetry of $Q(n+1)$ guarantees, however, that it will also lie in $B_{2^{\prime \prime} 2^{\prime}}$ - that is, there is a non-zero entry in $\bar{B}$ to the left of each diagonal entry in $B_{2^{\prime \prime} 2^{\prime \prime}}$. This is why we can apply the argument here to the 1'-rows, but not to the $1^{\prime}$-columns.]

We have established, then, that the rank of the following matrix is the same as the rank of $B_{33}$ :

$$
\left[\begin{array}{lll}
B_{1^{\prime} 2^{\prime}} & B_{1^{\prime} 2^{\prime \prime}} & B_{1^{\prime} 3} \\
B_{1^{\prime \prime 2} 2^{\prime}} & B_{1^{\prime \prime} 2^{\prime \prime}} & B_{1^{\prime \prime} 3} \\
B_{32^{\prime}} & B_{32^{\prime \prime}} & B_{33}
\end{array}\right] .
$$

We now establish that each of the blocks $B_{1^{\prime} 2^{\prime}}, B_{1^{\prime} 2^{\prime \prime}}, B_{1^{\prime \prime} 2^{\prime}}$, and $B_{1^{\prime \prime} 2^{\prime \prime}}$ consists only of zeroes. For each diagonal entry $q_{j j}$ in $B_{33}$ we can say only [because blocks $B_{31^{\prime}}, B_{31^{\prime \prime}}, B_{2^{\prime} 3}$, and $B_{2^{\prime \prime} 3}$ do not appear in (4.17)] that there will be (in the matrix of (4.17)) a non-zero entry above $q_{j j}$ in column $j$ or a non-zero entry to the left of $q_{j j}$ in row $j$. This turns out to be enough, however: each of the 2 - and 2"-columns in (4.17) must (by the same argument used above) be a linear combination only of those 3-columns that have no non-zeroes above the diagonal - and thus each of the blocks $B_{1^{\prime} 2^{\prime}}, B_{1^{\prime} 2^{\prime \prime}}, B_{1^{\prime \prime} 2^{\prime}}$, and $B_{1^{\prime \prime} 2^{\prime \prime}}$ consists only of zeroes.

Finally, notice that the blocks $B_{1^{\prime} 3}, B_{1^{\prime \prime} 3}, B_{32^{\prime}}$, and $B_{32^{\prime \prime}}$ in $Q(n)$ consist only of zeroes; thus, all non-zero entries in these blocks that appear in $Q(n+1)$ are also nonzero in $Q^{n+1}$. Suppose that at least one of the two blocks $B_{1^{\prime} 3}$ or $B_{1^{\prime \prime} 3}$ contains a non-zero, and that at least one of the two blocks $B_{32^{\prime}}$ or $B_{32^{\prime \prime}}$ contains a non-zero. Then Lemma A.3 guarantees that there is also a non-zero entry in one of the blocks $B_{1^{\prime} 3}, B_{1^{\prime \prime} 3}, B_{32^{\prime}}$, or $B_{32^{\prime \prime}}$, which we have just shown cannot be so. Thus, either

$$
B_{32} \text {, and } B_{32} \text { " both consist only of zeroes, }
$$

or else

$$
B_{1^{\prime} 3} \text { and } B_{1^{\prime \prime} 3} \text { both consist only of zeroes. }
$$

This establishes that every admissible $Q(n+1)$ has either the form (4.13) or (4.14), but allows that some have one form and some the other [i.e. it allows that some matrices $Q(n+1)$ satisfy (4.18) but not (4.19), and that some others satisfy (4.19) but not (4.18)]. However, if there is a $Q(n+1)$ - and thus a $Q^{n+1}$ - that violates (4.18) and another that violates (4.19), then convexity of the set of admissible matrices guarantees that there is a $Q(n+1)$ that violates both (4.18) and (4.19), which we have just shown cannot happen. Thus, either every admissible $Q(n+1)$ has the form (4.13), or else every admissible $Q(n+1)$ has the form (4.14).

\section{A potential application to externalities and decentralization}

Hurwicz, in his celebrated 1960 paper that introduced the formal analysis of allocation mechanisms, identificd a property of economic environments that he call- 
ed 'decomposability', ${ }^{3}$ the property by which he gave formal expression to the notion of an economic externality. Hurwicz's definition was later used by Ledyard (1971) to study the interplay between externalities, information, and Pareto optimality.

It is possible to model economic situations in such a way that Hurwicz's (1960) definition becomes a special case of the decomposability defined in the present paper (see Hurwicz, 1960, Sections 2 and 5). It therefore seems natural to continue interpreting indecomposabilities as externalities. Indeed, our interest in externalities arises from the belief that they are a primary cause of market failure, and Hurwicz and Walker (1988) obtain just such a 'failure' result, in spades: when externalities (indecomposabilities) are present, no decentralized mechanism will yield satisfactory outcomes via non-strategic behavior on the part of the mechanism's participants.

There is one respect in which this result - that externalities pose serious problems for decentralized economic decision-making - is at odds with the conventional wisdom: Hurwicz and Walker (1988) show that the classical pure exchange ('Edgeworth box') problem is naturally representable as an indecomposable indifference structure (in other words, an externality is present in the pure exchange problem); but the conventional view is that the pure exchange problem is the benchmark case representing complete absence of externalities.

I believe this clash with the conventional view, however, is a strength of the new notion of decomposability, not a weakness. It is only when the economy is 'atomistic' (there are so many agents that each is negligible) that decentralized markets are supposed to be fully successful. Perhaps, as the Hurwicz and Walker (1988) results indicate, when individuals are not negligible the benchmark case of pure exchange is (at least qualitatively) no more amenable to decentralization than those economic situations in which externalities of a more traditional kind are present. (See Groves and Ledyard, 1988, especially Section V, for a discussion of this.) The new notion of decomposability presented in the present paper may provide a framework in which it is possible to isolate the special nature of the "pure exchange externality' that causes it to lose its force when there are many individuals, while other kinds of externalities do not vanish - and perhaps even grow worse - when they involve many individuals.

\section{Acknowledgements}

Discussions with Richard McLean and Tom Muench were extremely helpful. This research was motivated by earlier work done with Leo Hurwicz, reported in Hurwicz and Walker (1988).

\footnotetext{
${ }^{3}$ Hurwicz attributed the term 'decomposability' to Jacob Marschak in fn. 11 of Hurwicz (1980).
} 


\section{Appendix A}

Lemma A.1. Let $A$ be a subspace of $\mathbb{R}^{m}$ and let $\mathscr{B}$ be a basis for $\mathbb{R}^{m} . A$ matrix is $A, \mathscr{B}$-admissible if and only if it can be written as $M^{\mathrm{t}} M$ for some matrix $M$ whose rows constitute (in the basis $\mathscr{B}$ ) a basis for $A$.

Proof. Let $\delta$ denote the dimension of $A$. We first show that the lemma is true for the usual basis $\mathscr{E}$, and then that is is also true for other bases $\mathscr{B}$.

Assume that the rows of $M$ form a basis for $A$ (in terms of the basis $\mathscr{E}$ ) and that $Q=M^{\mathrm{t}} M$. We must show that $Q$ is symmetric, that its column space is $A$, and that it is positive definite on $A \backslash\{0\}$. Writing $Q_{r s}$ for the entry in row $r$ and column $s$, we have:

$$
Q_{r s}=\sum_{k=1}^{\delta} M_{r k}^{\mathrm{t}} M_{k s}=\sum_{k=1}^{\delta} M_{k r} M_{k s}=Q_{s r} .
$$

Thus, $Q$ is symmetric. It is also clear from (A.1) that each row $Q_{r}$. of $Q$ is the linear combination of rows of $M$ in which the coefficients of the linear combination are the entries in column $r$ of $M$. In particular, then, every row (and, by symmetry, every column) of $Q$ is a member of $A$ - i.e. the column space of $Q$ is a subspace of $A$. Note also that a vector $x$ is a member of $I=d_{\text {def }} A^{\perp}$ if and only if $M_{r} x=0$ for every row $M_{r}$. of $M$. Finally, we have:

$$
x^{\mathrm{t}} Q x=x^{\mathrm{t}} M^{\mathrm{t}} M x=(M x)^{\mathrm{t}}(M x)=\sum_{r=1}^{\delta}\left(M_{r} \cdot x\right)^{2},
$$

which guarantees that $x^{t} Q x>0$ for $x \in A \backslash\{0\}$ (because $x \in A \backslash\{0\}$ implies that $x \notin I$, so that, as just shown above, $M_{r} . x \neq 0$ for some row of $M$ ) and which guarantees also that the rank of $Q$ is $\delta(=\operatorname{dim} A)$, so that the column space of $Q$ (which was shown above to be a subspace of $A$ ) has the same dimension as $A$-i.e. $A$ is in fact the column space of $Q$, as required. Thus, we have shown that if $Q$ is expressible as $M^{\mathrm{t}} M$, where the rows of $M$ constitute a basis for $A$, then $Q$ is admissible.

Now assume, conversely, that $Q$ is $\mathscr{E}$-admissible. Since $Q$ is symmetric, there is a non-singular maltix $P$ such that $P^{\dagger} Q P=D$, where $D$ is the diagonal matrix with entries $D_{r r}=1$ for $r \leq \delta$ and all other entries zero (the fact that all diagonal entries are non-negative is a result of the positive semidefiniteness of $Q$ ). But then $\left(P^{-1}\right)^{\mathrm{t}} D P^{-1}=Q-$ i.e. $M^{\mathrm{t}} M=Q$, where $M$ is the matrix consisting of the first $\delta$ rows of $P^{-1}$. Now $Q x=M^{t} M x$, so that every member of the column space of $Q$ is cxpressiblc as a linear combination of the columns of $M^{\mathrm{t}}$ (i.e. of the rows of $M$ ); and since $P^{-1}$ is non-singular, the rows of $M$ are linearly independent. In other words, the rows of $M$ form a basis for the column space of $Q$, which is the space $A$. This completes the proof of the lemma for the basis $\mathscr{E}$.

Now we must show that the lemma is true for an arbitrary basis $\mathscr{B}$. First we assume that $S=H^{t} H$ for some matrix $H$, the rows of which form (with respect to 
$\mathscr{B}$ ) a basis for $A$. Let $Q=C^{t} S C$, where $C$ is the matrix whose rows are the members of $\mathscr{B}$ (expressed in terms of $\mathscr{E}$ ), and let $M={ }_{\text {def }} H C$, so that the rows of $M$ form a basis (with respect to $\mathscr{E}$ ) for $A$. Then

$$
Q=C^{\mathrm{t}} H^{\mathrm{t}} H C=(H C)^{\mathrm{t}}(H C)=M^{\mathrm{t}} M,
$$

so that $Q$ is $\mathscr{E}$-admissible, and

$$
\tilde{Q}=\left(P^{-1}\right)^{\mathrm{t}} Q P^{-1}=\left(P^{-1}\right)^{\mathrm{t}} P^{\mathrm{t}} H^{\mathrm{t}} H P P^{-1}=H^{\mathrm{t}} H=S,
$$

so that $S$ is $\mathscr{B}$-admissible.

Conversely, assume that a given matrix $\tilde{Q}$ is $\mathscr{B}$-admissible - that is, $\tilde{Q}=$ $\left(P^{-1}\right)^{t} Q P^{-1}$ for some $\mathscr{E}$-admissible matrix $Q$. We must show that $\tilde{Q}$ can be written as $H^{\mathrm{t}} H$, where the rows of $H$ form (with respect to $\mathscr{B}$ ) a basis for $A$. Because the lemma is true for $\mathscr{E}$, we have $Q=M^{\mathrm{t}} M$, where the rows of $M$ form (with respect to $\mathscr{E})$ a basis for $A$. Let $H=M P^{-1}$. Since $M=H P$, each row of $H$ is the $\mathscr{B}$-expression of the corresponding row of $M$; in particular, then, each row of $H$ is the $\mathscr{B}$ expression of a member of $A$. Since the rows of $H$ are also linearly independent (because $P^{-1}$ is non-singular), they form a basis for $A$. Finally,

$$
\tilde{Q}=\left(P^{-1}\right)^{\mathrm{t}} Q P^{-1}=\left(P^{-1}\right)^{\mathrm{t}} M^{\mathrm{t}} M P^{-1}=\left(M P^{-1}\right)^{\mathrm{t}}\left(M P^{-1}\right)=H^{\mathrm{t}} H,
$$

so that $\bar{Q}$ is indeed expressible as $H^{\mathrm{t}} H$ for a matrix $H$ whose rows are a basis for $A$.

Lemma A.2. Let $Q$ be $A, \mathscr{B}$-admissible. If $q_{r s} \neq 0$, then $q_{r r} \neq 0$ and $q_{s s} \neq 0$.

Proof. Let $Q=M^{\mathrm{t}} M$, where the rows of $M$ are a basis (in $\mathscr{B}$ ) for $A$. It is clear from (A.1) that if $q_{r s} \neq 0$, then there is some $k$ for which $m_{k r}$ and $m_{k s}$ are both nonzero, and (A.1) thus yields $q_{r r}$ and $q_{s s}$ non-zero.

Lemma A.3. Let $Q$ be $A ; \mathscr{B}$-admissible. If both $q_{r r}$ and $q_{s s}$ are non-zero, then there is an $A, \mathscr{B}$-admissible matrix $Q^{\prime}$ for which $q_{r s}$ is non-zero.

Proof. Let $Q=M^{t} M$, where the rows of $M$ are a basis (in $\mathscr{B}$ ) for $A$. It is clear from (A.1) that $q_{r r} \neq 0$ implies that some row of $M$ (say, $M_{R}$.) has $m_{R r} \neq 0$. Similarly, some row $M_{S}$, , has $m_{S s} \neq 0$, since $q_{s s} \neq 0$. Obtain $M^{\prime}$ from $M$ by replacing row $M_{R}$. with $\alpha M_{R}$. $+\beta M_{S}$., where $\alpha$ and $\beta$ will be specified in a moment - i.e. for $r \neq R$, let $M_{r .}^{\prime}=M_{r}$, and let $M_{R .}^{\prime}=\alpha M_{R} .+\beta M_{S}$. . Let $Q^{\prime}=\left(M^{\prime}\right)^{\mathrm{t}} M ; Q^{\prime}$ is $A, \mathscr{B}$ admissible if $\alpha=0$, because the rows of $M^{\prime}$ span the same space as do the rows of $M$. The rows $Q_{\varrho}^{\prime}$. of $Q^{\prime}$ are as follows:

$$
\begin{aligned}
Q_{Q}^{\prime} & =\sum_{k=1}^{\delta} m_{k \varrho}^{\prime} M_{k}^{\prime} . \\
& =\sum_{k \neq R} m_{k \varrho} M_{k} .+m_{R \varrho}^{\prime} M_{R}^{\prime} .
\end{aligned}
$$




$$
\begin{aligned}
& =\sum_{k \neq R} m_{k \varrho} M_{k}+\left(\alpha m_{R \varrho}+\beta m_{S \varrho}\right)\left(\alpha M_{R}+\beta M_{S .}\right) \\
& =\sum_{k=1}^{\delta} m_{k \varrho} M_{k}+\left(\alpha^{2}-1\right) m_{R \varrho} M_{R}+\beta m_{S \varrho} M_{S \varrho}+\alpha \beta\left(m_{R \varrho} M_{S .}+m_{S \varrho} M_{R} .\right) \\
& =Q_{\varrho}+\left(\alpha^{2}-1\right) m_{R \varrho} M_{R}+\beta^{2} m_{S \varrho} M_{S .}+\alpha \beta\left(m_{R \varrho} M_{S .}+m_{S \varrho} M_{R} .\right)
\end{aligned}
$$

Thus,

$$
q_{\varrho \sigma}^{\prime}=q_{\varrho \sigma}+\left(\alpha^{2}-1\right) m_{R \varrho} m_{R \sigma}+\beta^{2} m_{S \varrho} m_{S \sigma}+\alpha \beta\left(m_{R \varrho} m_{S \sigma}+m_{S \varrho} m_{R \sigma}\right) .
$$

If there is a row $M_{\xi}$. of $M$ in which both $m_{\varrho r}$ and $m_{\xi s}$ are non-zero, then let $R=\xi$ and let $\beta=0$; then (A.3) yields $q_{r s}^{\prime} \neq q_{r s}$. If there is no such row, then choose $R$ and $S$ to satisfy $m_{R s}=m_{S r}=0$ (therefore, $m_{R r}$ and $m_{S s}$ are both non-zero), and choose both $\alpha$ and $\beta$ non-zero; then (A.3) yields:

$$
q_{r s}^{\prime}=q_{r s}+\alpha \beta m_{R r} m_{S s} \neq q_{r s} .
$$

It is useful to notice that when $Q=M^{\mathrm{t}} M$, each of the columns $Q . s$ of $Q$ is the linear combination of the columns of $M^{\mathrm{t}}$ in which the coefficients are the entries in columns $s$ of $M^{t}$, and also that if we rearrange the rows of $M$ (i.e. we keep the same basis for $A$, but we reorder it), we leave $Q$ unchanged.

\section{Appendix B}

In this appendix we derive some elementary results concerning perturbations of the diagonal entries of square matrices. Throughout the appendix, $n$ is an arbitrary integer $(n>1), A$ is an $n \times n$ matrix with entries $a_{r s}, \delta$ is an $n$-tuple of real numbers, and $D(\delta)$ is the $n \times n$ diagonal matrix whose diagonal entries are $\delta_{1}, \ldots, \delta_{n}$.

Lemma B.1. If $a_{11} \neq 0$, then there are arbitrarily small values of $\delta_{2}, \ldots, \delta_{n}$ for which $A+D(\delta)$ is non-singular, where $\delta_{1}=0$.

Proof. The lemma is clearly true for $n=2:|A+D(\delta)|=a_{11} a_{22}-a_{12} a_{21}+a_{11} \delta_{2}$. We assume, inductively, that the lemma is true for $n-1$, and we will show that it is true for $n$.

Let $\delta^{\prime}$ denote $\left(\delta_{1}, \ldots, \delta_{n-1}\right)$. Expanding $|A+D(\delta)|$ by the cofactors of its rightmost column, and denoting those cofactors by $C_{r n}\left(\delta^{\prime}\right)$ for rows $r=1, \ldots, n$, we have:

$$
|A+D(\delta)|=\left(a_{n n}+\delta_{n}\right)\left|A_{n-1}+D_{n-1}\left(\delta^{\prime}\right)\right|+\sum_{r=1}^{n-1} a_{r n} C_{r n}\left(\delta^{\prime}\right)
$$

where $A_{n-1}$ and $D_{n-1}$ are the submatrices of $A$ and $D$ formed by deleting the $n$th row and column of each. Because the lemma is true for $n-1$, we can choose $\delta^{\prime}$ in such a way that $\delta_{1}=0$ and $\left|A_{n-1}+D_{n-1}\left(\delta^{\prime}\right)\right| \neq 0$. If this does not yield $|A+D(\delta)| \neq 0$, then we let $\delta_{n} \neq 0$, which succeeds because $\delta^{\prime}$ is independent of $\delta_{n}$ in (B.1). 
Lemma B.2. Let $A$ be non-singular, let $c$ be a column vector, and whenever the matrix $A+D(\delta)$ is non-singular, let $y(\delta)$ be the (unique) solution of the equation $(A+D(\delta)) y(\delta)=c$. If $c_{1} \neq 0$, then there are arbitrarily small values of $\delta$ for which $\delta_{1}=0$ and $y_{1}(\delta) \neq 0$ - i.e. the first component of the solution vector can be made non-zero by arbitrarily small perturbations of the last $n-1$ diagonal entries of the matrix $A$.

Proof. This is an immediate corollary of Lemma B.1, obtained by using Cramer's Rule to express the solution vector $x(\delta)$. (Note that $A+D(\delta)$ is nonsingular for a neighborhood of $\delta=0$.)

Lemma B.3. Let $A$ be non-singular, and let $b$ and $c$ be arbitrary column vectors. Let $y=A^{-1} c$, let $x=A^{-1} b$, and whenever the matrix $A+D(\delta)$ is non-singular, let $x(\delta)$ be the (unique) solution of the equation $(A+D(\delta)) x(\delta)=b$ and let $f(\delta)=c_{t} x(\delta)$. Then the partial derivative of $f$ at $\delta=0$ with respect to $\delta_{1}$ is $-x_{1} y_{1}-i$.e.

$$
\frac{\partial f}{\partial \delta_{1}}(\mathbf{0})=-x_{1} y_{1}
$$

Thus, in particular, if both $x_{1}$ and $y_{1}$ are non-zero, then the scalar product $c^{\mathrm{t}} x$ can be perturbed by making arbitrarily small perturbations of the diagonal entry $a_{11}$ of $A$.

Proof. Let $x^{*}=\left(x_{1}, 0, \ldots, 0\right)^{\mathrm{t}}$. Application of the Implicit Function Theorem to the equation $(A+D(\delta)) x(\delta)=b$ yields:

$$
\left[\frac{\partial x_{j}}{\partial \delta_{1}}\right]_{j=1}^{n}=A^{-1} x^{*}
$$

Thus, at $\delta_{1}=0$ we have:

$$
\frac{\partial f}{\partial \delta_{1}}=\sum_{1}^{n} c_{j} \frac{\partial x_{j}}{\partial \delta_{1}}=c^{\mathrm{l}} A^{-1} x^{*}=y^{\mathrm{t}} x^{*}=-x_{1} y_{1}
$$

\section{References}

T. Groves and J. Ledyard, Incentive compatibility since 1972, in: T. Groves, R. Radner, and S. Reiter, eds., Information, Incentives, and Economic Mechanisms (University of Minnesota Press, 1988).

L. Hurwicz, Optimality and informational efficiency in resource allocation processes, in: K. Arrow, S. Karlin, and P. Suppes, eds., Mathematical Methods in the Social Sciences (Stanford University Press, 1960), ch. 3.

L. Hurwicz and M. Walker, On the generic non-optimality of dominant-strategy allocation mechanisms: A general theorem that includes pure exchange economies, Econometrica, forthcoming (1989).

J. Ledyard, The relation of optima and market equilibria with externalities, J. Economic Theory 3 (1971) 54-65. 